\title{
Concept-based model explanations for Electronic Health Records
}

\author{
Diana Mincu \\ Google Research \\ London, UK \\ Sebastien Baur
Google Health
London, UK \\ Anne Mottram \\ DeepMind \\ London, UK
}

\author{
Eric Loreaux \\ Google Health \\ Palo Alto, CA, USA \\ Ivan Protsyuk \\ Google Health \\ London, UK
Nenad Tomasev
Deepmind
London, UK
Jessica Schrouff*
Google Research
London, UK
schrouff@google.com

\author{
Shaobo Hou \\ DeepMind \\ London, UK
Martin Seneviratne
Google Health
London, UK
Alan Karthikesalingam
Google Health
London, UK

\begin{abstract}
Recurrent Neural Networks (RNNs) are often used for sequential modeling of adverse outcomes in electronic health records (EHRs) due to their ability to encode past clinical states. These deep, recurrent architectures have displayed increased performance compared to other modeling approaches in a number of tasks, fueling the interest in deploying deep models in clinical settings. One of the key elements in ensuring safe model deployment and building user trust is model explainability. Testing with Concept Activation Vectors (TCAV) has recently been introduced as a way of providing humanunderstandable explanations by comparing high-level concepts to the network's gradients. While the technique has shown promising results in real-world imaging applications, it has not been applied to structured temporal inputs. To enable an application of TCAV to sequential predictions in the EHR, we propose an extension of the method to time series data. We evaluate the proposed approach on an open EHR benchmark from the intensive care unit, as well as synthetic data where we are able to better isolate individual effects.
\end{abstract}

\section{CCS CONCEPTS}

- Computing methodologies $\rightarrow$ Artificial intelligence; Machine learning; Neural networks.

\section{KEYWORDS}

Explainability, time series, human-understandable concepts, electronic health records (EHR)

${ }^{*}$ Corresponding author.

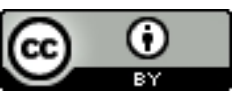

This work is licensed under a Creative Commons Attribution International 4.0 License.

ACM CHIL '21, April 8-10, 2021, Virtual Event, USA

(C) 2021 Copyright held by the owner/author(s).

ACM ISBN 978-1-4503-8359-2/21/04.

https://doi.org/10.1145/3450439.3451858
ACM Reference Format:

Diana Mincu, Eric Loreaux, Shaobo Hou, Sebastien Baur, Ivan Protsyuk, Martin Seneviratne, Anne Mottram, Nenad Tomasev, Alan Karthikesalingam, and Jessica Schrouff. 2021. Concept-based model explanations for Electronic Health Records. In ACM Conference on Health, Inference, and Learning (ACM CHIL '21), April 8-10, 2021, Virtual Event, USA. ACM, New York, NY, USA, 11 pages. https://doi.org/10.1145/3450439.3451858

\section{INTRODUCTION}

Wider availability of Electronic Health Records (EHR) has led to an increase in machine learning applications for clinical diagnosis and prognosis [e.g., 1, 2]. Larger de-identified datasets and public benchmarks have fueled the application of increasingly complex techniques such as recurrent neural networks (RNNs) to predict adverse clinical events [e.g., 6, 16, 23, 29, 31]. RNNs can operate over a sequence of health information, iteratively combining input data with internal memory states to generate new states, making them suitable for continuous clinical predictions. While these memorystoring networks allow for accurate and dynamic predictions, it is often difficult to examine the mechanism by which clinical information is being translated into outputs. In healthcare, as in other fields in which trust is paramount, it is not sufficient to show state of the art discriminative performance; clinicians have also deemed it critical that models provide local and global explanations for their behavior [30].

Multiple approaches have been proposed to provide explanations for machine learning models applied to EHR data [see 19, for a review], with a focus on attention-based methods when the architecture relies on RNNs [e.g., 3, 22, 23]. Typically, those interpretability techniques ranks the input features based on their attention scores. However, single feature rankings might not highlight clinical states that encompass multiple input features (e.g. "infection") in an intuitive manner. To address this issue of human understandability, Panigutti et al. [18] use an ontology of diagnoses to provide insights across single features. This approach, however, 
relies on diagnoses which are typically recorded at the end of an admission and is therefore not suitable to identify temporal changes across features that reflect a clinical concept, nor is it able to provide continuous predictions.

On the other hand, human-understandable explanations have been successfully developed for computer vision applications: Testing with Concept Activation Vectors [TCAV, 15] relies on humanunderstandable "concepts" to derive model explanations. Practitioners or end users can select examples from the data that embody intuitive concepts (e.g. "pointy ears" or "stripes"), and these examples are then used to map concepts to the model's activation space in the form of concept activation vectors (CAVs). CAVs can then be used to provide global explanations, as well as assess the presence or absence of a concept in local examples.

In this work, we define "clinical concepts" from temporal EHR input features to improve the human-understandability of posthoc explanations of continuous clinical predictions. Our approach leverages TCAV [15] and can be applied to previously trained models without restrictions on model inputs or RNN architecture. Our contributions are as follows:

- We extend the TCAV approach to the time series setting by defining metrics assessing (1) whether the model encodes the concept, (2) whether the concept is "present" in examples, and (3) whether a concept influences the model's predictions.

- We design a synthetic time series dataset to evaluate (conceptbased) attribution methods and demonstrate that the proposed technique is faithful.

- We propose a framework to define human-understandable concepts in EHR and illustrate it using the de-identified MIMIC-III benchmark dataset [12].

\section{METHODS}

Notation: We consider a set of multivariate time series $\mathcal{X}:=$ $\left(x_{i, t, d}\right)_{i \leq N, t \leq T_{i}, d \leq D}$, where $x_{i, t, d} \in \mathbb{R}, N$ is the number of time series (i.e. patients), $D$ is the number of features per time step and $T_{i}$ the number of time steps for patient $i$. We define $\mathbf{x}_{d}$ as the time series for feature $d \in\{1, \ldots, D\}$ for a single example. The label $\mathbf{y} \in\{0,1\}^{N \times T}$ exists for all examples and all time steps. We train a recurrent neural network $F: \mathcal{X} \rightarrow[0,1]^{T}$ with $L$ layers. For a given layer $1 \leq l \leq L$ and time step $1 \leq t \leq T$, we can write the predicted output of $F$ as $F_{t}(\vec{x}):=h\left(f_{l}\left(\vec{x}_{1: t}\right)\right)$ where $f_{l}\left(\vec{x}_{1: t}\right)$ is the activation vector at the $l$-th layer after $t$ time steps, further referred to as $\vec{a}_{t, l}$ and $h$ represents the operations in layers $l \ldots L$. Please note that we consider binary classification settings, but the approach extends to multi-class predictions.

\subsection{Concept-based explanations over time}

In this section, we extend TCAV [15] to account for the temporal dimension. TCAV relies on two main steps: (1) Building a concept activation vector (CAV) for each concept, and (2) assessing how the concept influences the model's decision.

Building a $C A V$ :. To build a CAV, Kim et al. [15] sample positive and negative examples for a concept, record their activations $\vec{a}_{l}$ at each layer $l$ of the network and build a linear classifier (e.g. logistic regression) distinguishing between activations related to positive and negative samples. To extend this approach to timeseries, we identify a 'time window of interest' $\left[t_{\text {start }}, t_{\text {end }}\right]$ that reflects a trajectory corresponding to a concept, i.e. during which some features or feature changes are present. We define a 'control' group as a set of trajectories in which the concept does not manifest. We then collect the model's activations from $t=t_{\text {start }}$ to the end of the window $t_{\text {end }}$ for both groups, and training data for CAV learning is defined based on three different strategies:

- $\mathrm{CAV}_{t_{\text {end }}}$ : we record the model's activations in each layer at $t_{\text {end }}$. This reflects the assumption that the trajectory can be represented by its end point.

- $\mathrm{CAV}_{t_{\text {start }}: t_{\text {end }}}$ : we record the model's activations at each time step between $t_{\text {start }}$ and $t_{\text {end }}$, using them as samples in the linear classifier. This approach hypothesizes that each time step in the trajectory represents a key component of the concept pattern.

- $\mathrm{CAV}_{t_{\text {end }}-t_{\text {start }}}$ : we record the model's activations at $t_{\text {start }}$ and at $t_{\text {end }}$ and use their difference to train the CAV. In this case, we assume that changes in activations represent the concept of interest.

A concept is considered as "encoded" in the model if the linear model performs significantly above chance level. We assess the linear classifier's performance using a bootstrap resampling scheme ( $\mathrm{k}=100$, stratified where relevant) and perform random permutations (1,000 permutations, 10 per bootstrap resampling) of the labels to obtain a null distribution of balanced accuracy and area under the receiver-operating curve (AUROC). We assess a CAV as significant if all metrics are higher than the estimated null distributions with $p<0.05$. We then estimate the generalizability of the classifier across time steps by performing the classification at all time points $(t=1, \ldots, T)$, where we give a label of 1 (resp. 0$)$ at time points where the concept is present (resp. absent), when that information is known (i.e. synthetic data), and a label of 1 (resp. 0) for all time points of concept (resp. control) time series if not known (i.e. clinical application). This measure of performance beyond the $\left[t_{\text {start }}, t_{\text {end }}\right]$ window allows to understand whether concepts are represented similarly across all time points in the sequence, or whether the signal is specific to the window selected.

Presence of the concept in a sample: The original TCAV work [15] computes the cosine similarity between the activations $\vec{a}_{l}$ of a sample and the obtained CAV at each layer to estimate how similar an image is to a concept. This similarity measure can be thought of as estimating whether a concept is manifesting or "present" in the sample. In time series, it can be computed at each time point independently to obtain a (local) trajectory of concept presence per layer:

$$
\operatorname{tCA}_{C}\left(\vec{x}_{t}\right)=\frac{\vec{a}_{t}^{T}}{\left\|\vec{a}_{t}\right\|_{2}} \vec{v}_{C}
$$

Where $\overrightarrow{v_{C}}$ corresponds to the unit norm CAV of concept $C$. This formulation can be extended to estimate whether the activations change over time in the direction of the concept by replacing $\vec{a}_{t}$ by $\left[\vec{a}_{t}-\vec{a}_{t-d t}\right]$ (following the assumption of local linearity in [15]), where $d t$ represents a constant lag in a time shifting window. This is relevant to investigate concepts that would vary across time, e.g. by 
becoming more severe, and this the formulation used throughout this work.

Influence of the concept on the model's prediction: Kim et al. [15] define the Conceptual Sensitivity (CS), to estimate how the model's gradients align with the CAV. This quantity, when aggregated over samples, represents a global explanation. Mathematically, CS can be computed as the directional derivative:

$$
\begin{aligned}
\operatorname{CS}_{C, l, t}\left(F, \vec{x}_{t}\right) & :=\frac{\partial h\left(f_{l}\left(\vec{x}_{t}\right)\right)}{\partial \vec{v}_{C}} \\
& =\nabla h\left(f_{l}\left(\vec{x}_{t}\right)\right)^{T} \vec{v}_{C}
\end{aligned}
$$

Which amounts to computing the cosine similarity between the direction of the CAV and the model's gradients. In the present case, CS is computed at every time step of the local trajectory by taking the gradients of the models w.r.t. the sigmoid of the logits. The obtained scores can be aggregated over time and/or over samples to obtain global concept attributions.

We believe that $t C A$ and CS can be seen as providing complementary information for global explanations, i.e. how is the presence/absence of the concept varying across time, and is the model influenced by the concept to make its decisions? Indeed, a concept being "present" does not guarantee that the model relies on it for prediction. On the other hand, a CS score of 0 means that affecting how present the concept is has (locally) no influence on the model output, but CS cannot reflect on whether the concept is present or absent.

\subsection{Synthetic timeseries}

Inspired by [8], we evaluate the proposed approach on a synthetic dataset designed to isolate individual effects.

Dataset design: In our setup, a concept $C$, akin to a latent variable, can manifest through a causal relationship with a time series' features and label (see Figure 1). For simplicity, we consider a binary behavior for $C$ : a concept either manifests and is "present" in a sample after a selected temporal "change point", or it is "absent". When present, each feature has a predefined likelihood $p(d=1 \mid C=$ $1)=p(d=0 \mid C=0)$ of exhibiting the concept's pattern, which can be any detectable change in behavior. If that likelihood is set to zero for a feature, the concept will not influence the feature's behavior. Similarly, the concept influences the label $y$ after the same change point with likelihood $p(y=1 \mid C=1)=p(y=0 \mid C=0)$. These parameters are set at the dataset level (see Supplement for the sampling algorithm).

In this work, we define two concepts, $C_{1}$ and $C_{2}$, and two corresponding labels, $y_{1}$ and $y_{2}$, influenced by $C_{1}$ and $C_{2}$ respectively with $p(y \mid C)=1$. We generate 10 numerical features with Gaussian background noise, and link $C_{1}$ and $C_{2}$ to non-overlapping sets of 5 features each. The pattern for all concept-activated features is the emergence of a sinusoid with fixed frequency and amplitude added to the Gaussian noise. The simplicity of this setup ensures that the ground truth is well understood.

We note that our code supports more complex settings, e.g. overlapping concept-feature space or multi-concept label contingency tables (see Supplement). In addition, feature/concept behaviors can

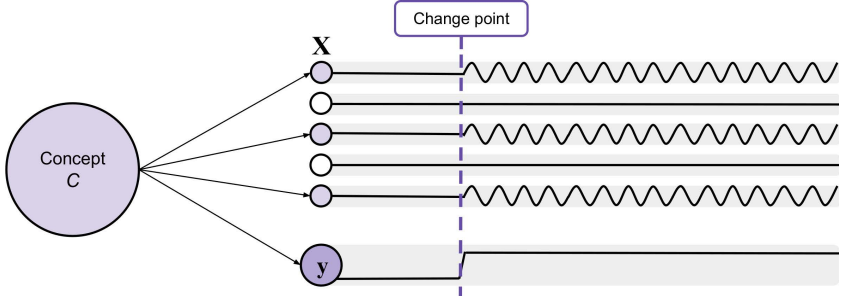

Figure 1: Illustration of the causal graph and sampling of time series for the synthetic dataset. A concept $C$ affects a subset of features $i \in 1,3,5$ and a label $\vec{y}$ after the 'change point'.

be made more realistic (e.g. by including binary variables). Therefore, this dataset is suitable for assessing attributions at both the feature and concept level, and could be used in other scenarios.

Model Training: The model consists of a 3-layer stacked LSTM [10] RNN, with 64 hidden units contained in each layer. These layers are followed by a fully connected layer. The model is trained using cross entropy loss with the Adam optimizer (fixed learning rate of $3 e-4)$ and batch size of 32, over 10000 randomly sampled batches. We report the model performance across all time steps and examples based on accuracy, AUROC and area under the precision-recall curve (AUPRC).

Concept definition: We use $C_{1}$ and $C_{2}$ as our concepts. We assign $t_{\text {start }}$ as the change point and define the 'time of interest' $t_{\text {end }}$ as 25 samples (arbitrary choice) after the change point, to ensure the concept is either present (concept group) or absent (control group). We randomly select 100 samples from the validation set to build a $\mathrm{CAV}$ for each concept (i.e. $C_{1}$ and $C_{2}$ ) and layer $l$. To ensure that the model is able to identify the concepts, we filter for a minimum model accuracy of 0.8 on a per-sequence basis (arbitrary threshold). The performance of each CAV is assessed on the held-out time steps during the bootstrap procedure, as well as on 500 other time series of the validation set (all time steps).

\subsection{Illustration on clinical predictions}

Data: We use the de-identified critical care EHR data from the Medical Information Mart for Intensive Care (MIMIC-III) [7, 11, 12] to investigate a real-world application of our technique. After filtering out patients under the age of 18 , the MIMIC-III dataset contained 47,296 patients, which were randomised across training (80\%), validation (10\%), and test (10\%) sets. Each patient's medical history is converted to a time series of one-hour aggregates including different structured data elements (medication, labs, vitals, ...) represented by numerical and binary variables [29, see Supplement for details]. Importantly, our data representation is sparse at each time step, and includes 32,170 continuous variables and 38,600 binary variables, for a total of 70,770 features.

Model: We focus on the predictions 48 hours in advance of an Acute Kidney Injury (AKI) event of stage 1 or more [max stage 3, as per the Kidney Disease Improving Global Outcomes classification, KDIGO, 14]. We use the same model architecture as described in [29] which consists in a 3-layer stacked RNN with 
residual connections and add dropout probability of 0.4 to the output connections of each LSTM cell. The model's hyper-parameters were defined based on a grid search on the validation set. The model's performance is then assessed on the test set using AUPRC given the low prevalence of AKI in the dataset. For comparison with the literature, we also report AUROC.

Concept definition: Based on clinical input, we define illustrative concepts by relying on rule-filtering of specific clinical events from patients included in the validation set. These events then serve to determine $t_{\text {start }}$ and $t_{\text {end }}$ for the different CAV building strategies. We define multiple concepts:

- 'AKI', that is directly related to the outcome labels for sanity check.

- 'Nephrotoxicity', a known risk factor for kidney injury.

- 'Antibiotics'. This concept aims at identifying bacterial infections, a proxy for sepsis, which is another known risk factor for kidney injury.

- 'Sex'.

The AKI concept group is defined as follows: admissions where the patient is recorded to have normal renal function [i.e. no AKI, based on the serum creatinine and the KDIGO criteria 14], and later in the admission renal function degrades to an AKI stage $2\left(t_{\text {end }}\right)$. The control group for the AKI concept is defined as: admissions where no AKI is recorded, with at least one normal creatinine measurement. In this case, a random one-hour bucket is selected as representing the end point of the trajectory, i.e. $t_{\text {end }}$. The AKI concept is purposefully circular to assess how the proposed approach scales to real-world problems.

To define the nephrotoxicity concept, we select admissions where the patient has normal renal function, then receives a particular type of nephrotoxic agent, which is followed by an AKI stage 1,2 or 3. Please note that we do not select admissions where the nephrotoxic agent caused the AKI, as we do not have that information. We selected one class of nephrotoxic drugs, non-steroidal antiinflammatory drugs (NSAIDs). The control group for this concept included admissions where the patient had normal renal function followed by an AKI (stage 1, 2 or 3), without receiving an NSAID medication before the adverse event ${ }^{1}$. This 'relative' concept [15] investigates whether the model encodes the differential effect of NSAIDs on AKI, compared to all other factors correlating with or causing AKI.

We identified 18 antimicrobial agents (see Supplement) and defined an 'antibiotics' concept to act as a proxy for detecting sepsis. The selection of admissions for CAV building is similar to that of the nephrotoxicity concept.

The 'sex' concept investigates whether self-reported sex affects the model's predictions, as the authors of [29] reported lower performance of the model on women compared to men. To this end, we build a CAV distinguishing between admissions of females and males leading to an AKI episode. We consider time windows spanning 12 hours before the AKI event, 24 hours before the AKI event

\footnotetext{
${ }^{1}$ Please note that we control for the endpoint of the trajectory $t_{\text {end }}$ to correspond to an AKI event. One could however control for the proportion of AKI samples across both groups. On the other hand, not controlling for the endpoint might introduce confounding factor if patients receiving NSAIDs have a higher prevalence of AKI.
}

or the beginning of the admission to the AKI event. Importantly, sex is not included as a feature in the model training.

To avoid potential confounding factors in the CAV, we use the same number of patients in the concept and control groups and choose patients to match on selected data statistics between groups. The features we match on are age, gender, duration of hospital admission, time between admission and AKI (or time between admission and selected $t_{\text {end }}$ for controls without AKI events), and inpatient mortality. For each of the patients in the concept and control groups we calculate the vector of features that we wish to match on, standardising based on the training dataset mean and standard deviation. From the pool of candidate examples for the control group, we then select those that minimise the total L1 distance between feature vectors in the control and concept group. The distance minimisation problem is solved using the Munkres algorithm [17]. We then select similar numbers of time steps within each patient (arbitrarily selected as 10), based on different sampling strategies: random sampling across the $t_{\text {start }}: t_{\text {end }}$ window, sampling at equal intervals with the interval being computed based on the number of time steps for a patient $T_{i}$, and sampling among the true positive predictions. We report results for sampling across equal intervals, and did not identify a significant effect of the sampling strategy on the results.

We build CAVs for each concept based on selected concept and control groups from the training set. We then compute $t C A$ and CS on patients from similarly selected groups from the test set.

Comparison with feature-based attributions: We present the results of occlusion [33] and gradient [25] analyses, computed for features that are 'present' in a time step. These attribution scores are estimated at each time step independently, as in [29, see Supplement for details]. The aim of these analyses is to highlight the differences between feature-based and concept-based techniques. We however believe that both can potentially be useful, and do not intend to recommend one over the other.

\section{RESULTS}

\subsection{Synthetic dataset}

Data and model: We generate 10,000 time series of 100 time points each to predict $y_{1}$ and $y_{2}$. After training, the model reaches 95.34\% accuracy, 0.8511 AUPRC and 0.9274 AUROC on a test set of 1,000 time series.

3.1.1 Building the CAV. The different strategies lead to significant CAVs for both $C_{1}$ and $C_{2}$ as assessed on held-out test sets, although $\mathrm{CAV}_{t_{\text {end }}-t_{\text {start }}}$ has relatively lower performance (see Figure $2 \mathrm{a}$ for $C_{1}$ and Supplement). All CAVs generalize to time points outside of the $\left[t_{\text {start }}: t_{\text {end }}\right]$ time window used for building, on further validation time series (Figure $2 \mathrm{~b}$ ). We however note that this result might be driven by the simplicity of our synthetic dataset and the high performance of the RNN model. For compactness, further results focus on the $\mathrm{CAV}_{t_{\text {start }}: t_{\text {end }}}$ strategy. All strategies however lead to similar results in terms of CS and $t C A$ scores.

3.1.2 Presence of the concept over time. $t C A$ is estimated at each time step, using a lag of 25 time steps (arbitrary choice) for both concepts. Figure $2 \mathrm{c}$ displays the average across aligned time series. 
$\mathbf{a}$
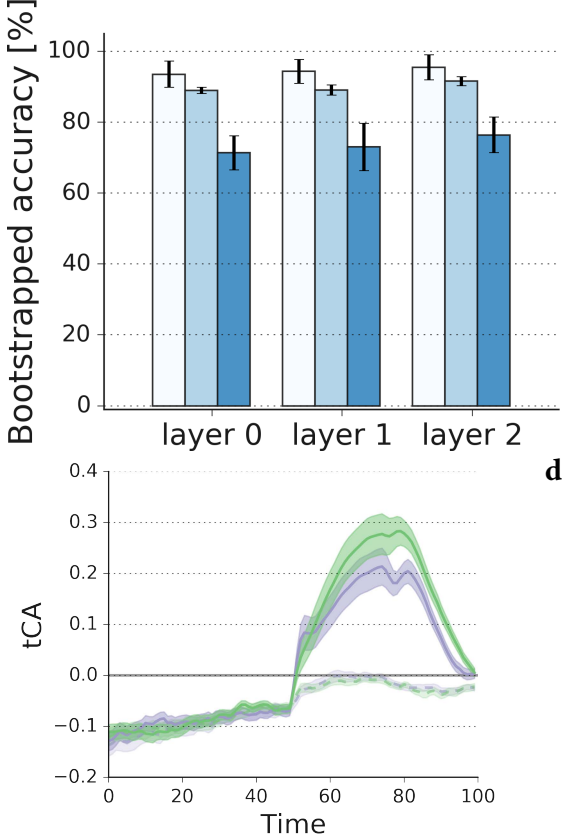

b
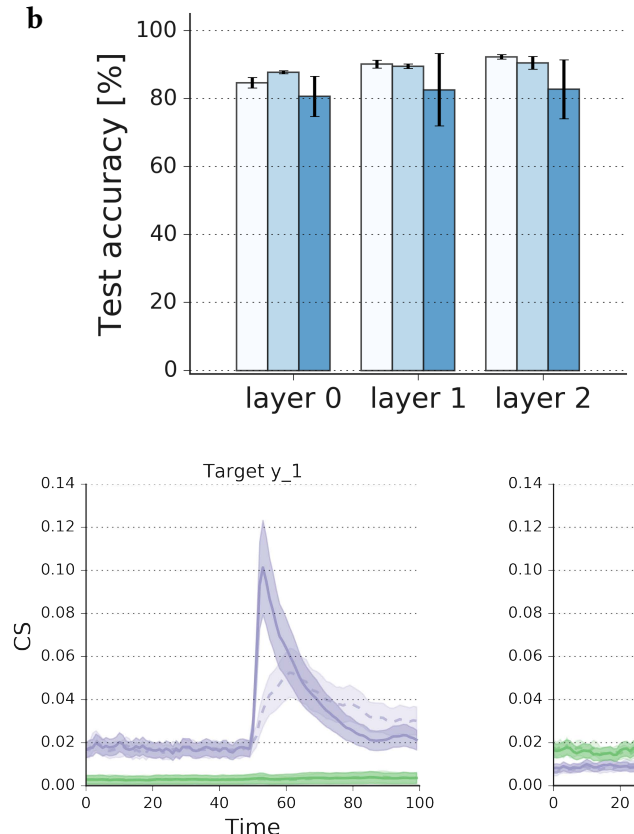

CAV strategy

$\square$ t_e

$\square$ t_s:t_e

$\square$ t_e-t_s

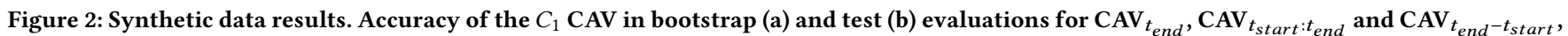
in \%. c $t C A$ scores (layer 2$)$ averaged across time series, and bootstraps (mean \pm std) when a concept ( $C_{1}$ green, $C_{2}$ purple) is absent (light shade, dotted line) or present (dark shade). All timeseries are aligned to have their changepoint at $t=50$. d CS scores for target $y_{1}$ (left) and $y_{2}$ (right).

We observe that $t C A$ has a negative score when the concept is absent, and then sharply transitions to positive scores when the concept becomes present $(t=50)$.

3.1.3 Influence of the CAV. We compute CS at each time point and display global trajectories of the obtained scores in Figure 2d. The results display the expected behavior: for target $y_{2}$, only concept $C_{2}$ has CS scores that are not tightly distributed around zero at all time points. In addition, CS scores are low before the change point (here aligned across all time series as $t=50$ ), reflecting the "absence" of $C_{1}$, while they become positive at the change point, when the label and concept manifest. These results are replicated for target $y_{1}$ and $C_{1}$ (see Supplement).

\subsection{MIMIC dataset}

3.2.1 Data and model. Our model predicts AKI of any severity within the next 48 hours with a AUPRC of 0.491 and AUROC of 0.798 . It is difficult to make direct comparisons with the literature as, to our knowledge, comparable continuous AKI predictions on MIMIC have not been reported on to date. However, there are a number of similar studies on different EHR datasets: Simonov et al. [24] report an AUROC of 0.74 for AKI within 24 hours using a discrete time logistic regression triggered after every new measurement; while Kate et al. [13] report up to 0.724 with a similar setup. Flechet et al. [5] predict AKI within the next 7 days in an ICU population with AUROC ranging from 0.80-0.95 depending on the window of input data.

\subsubsection{AKI concept.}

Building the CAV:. The AKI concept was built using 161 inpatient episodes selected from the validation set. We tested different building strategies, namely $\mathrm{CAV}_{t_{\text {end }}}$ with $t_{\text {end }}$ being the time of AKI, $\mathrm{CAV}_{t_{\text {start }}: t_{\text {end }}}$ using time steps included in the 12 or 24 hours before $\mathrm{AKI}$ and $\mathrm{CAV}_{t_{\text {end }}-t_{\text {start }}}$ by subtracting the activation at time of admission from that of the time of AKI. The obtained linear classifier was then evaluated on examples selected from the test set, on the same points as used for training (i.e. $t_{\text {end }}$, last 12 hours, ...) as well as on all other time points. We observe high training accuracy for all models, as evaluated per the bootstrap scheme (Figure 3a (left), Supplement). When evaluating on the test examples, we observe high accuracy on the equivalent time steps as used for training. This accuracy decreases for the concept group when evaluating on test time series (all time steps), with $\mathrm{CAV}_{t_{\text {end }}}$ and $\mathrm{CAV}_{t_{\text {end }}-t_{\text {start }}}$ seemingly overfitting to the training time steps (Figure 3a (right)). This result suggests that time steps outside of the selected window might not reflect the same signals as time steps within the selected window.

Presence of the concept over time: We select the model with best generalization across time steps to compute the alignment, i.e. using the last 24 hours before AKI and compute $t C A$ with a lag $d t$ of 24 hours. We present the results using the test examples at time 48 hours before $t_{\text {end }}$ (our prediction horizon, $t_{0}$ on Figure $\left.3 \mathrm{~b}(\mathrm{left})\right)$ and at time $t_{\text {end }}$ (corresponding to time of AKI stage 2 for the concept group and a random no AKI event for the control group, $t_{1}$ ).

As previously observed on the synthetic data, using the difference in activations on a 24 hour sliding window leads to higher $t C A$ scores when the concept is present compared to when it is absent, with an increase from $t_{\text {start }}$ to $t_{\text {end }}$. We plot the time series of 

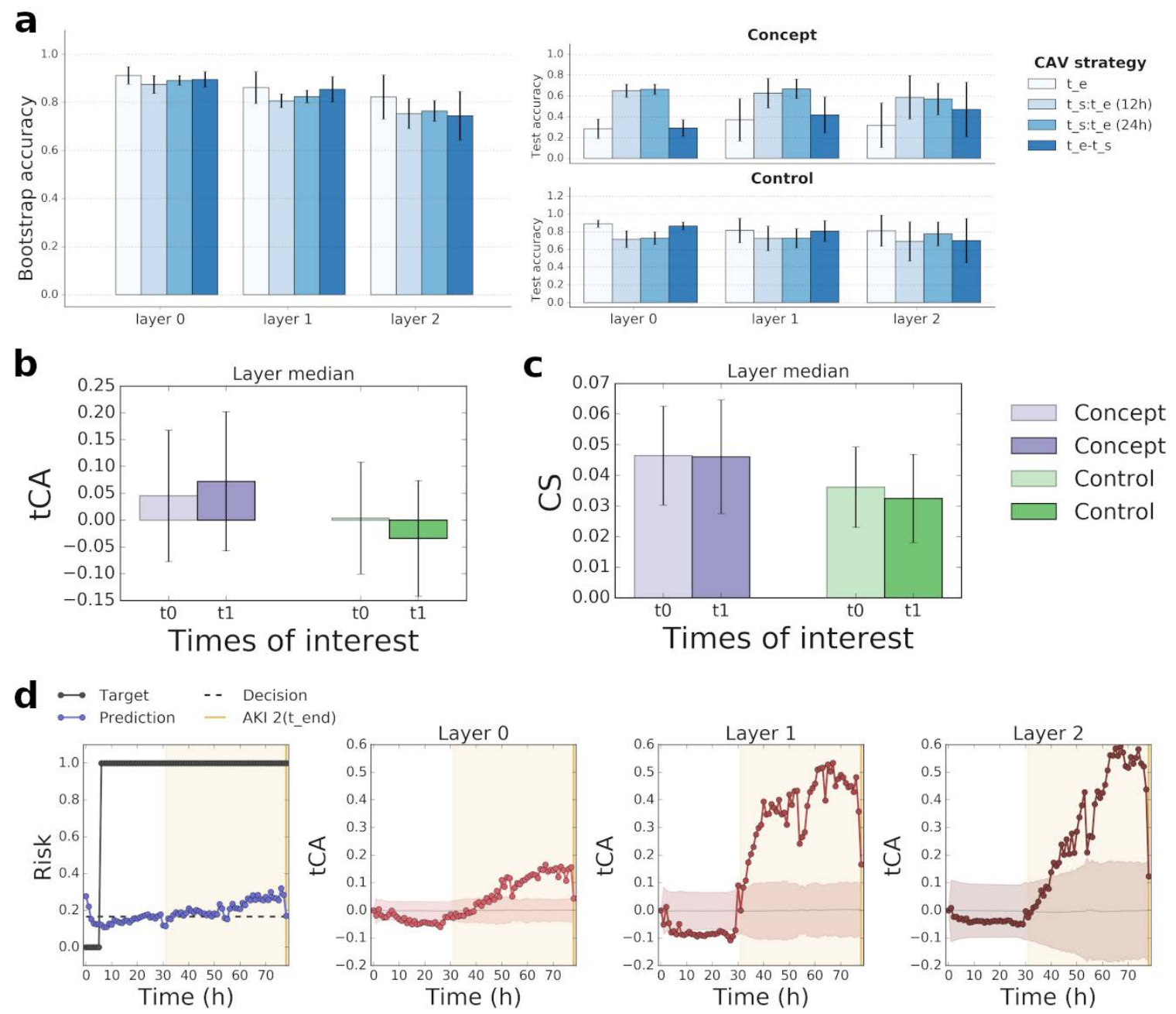

Figure 3: MIMIC results, AKI concept. a CAV linear model performance on held-out samples (left) and on test samples for concept (top) and control (bottom) samples. Each bar represents a CAV building strategy. $b t C A$ global scores, averaged across patients (mean \pm std) for the concept (purple) and control (green) groups 48 hours before AKI $\left(t_{0}\right)$ and at time of AKI stage $2\left(t_{1}\right)$. c $C S$ scores. $d$ Single patient timeseries, displaying the label (AKI 1+ within 48h) and model's output, as well as $t C A$ for each layer, and its null hypothesis (shaded). The yellow shaded area represents the prediction horizon of the model, i.e. within 48 hours of AKI 2.

concept alignment for an example patient in Figure $3 \mathrm{~d}$ for each layer of the model, along with the distribution of $t C A$ scores when using permuted CAV vectors (i.e. built from randomized concept/control labels) for an example from the evaluation group with AKI. The $t C A$ scores per time step seem to reflect the risk as predicted by the model: the $t C A$ score starts increasing around time $t 30$, i.e. around 48 hours before the AKI stage 2 event detected by the KDIGO label.

Influence of the $C A V$ :. We observe a positive influence of the CAV on the target, as displayed by strictly positive values of CS on the different samples and time points considered (Figure 3c). This is expected due to the circularity in the definition of this concept.
Building the CAV:. Similarly to the AKI concept, we train three variants of the linear classifier ${ }^{2}$, where we use all samples between 24 hours before AKI to the time of AKI $\left(t_{\text {end }}\right)$, all samples between the time of NSAIDs and the time of AKI, or the difference in activation between the time of AKI and the time of NSAIDs. While all classifiers are assessed as significant per non-parametric permutation testing, model performance is overall lower than for the AKI concept both for the held-out and validation time steps (Figure $4 \mathrm{a}$ and Supplement). As previously, we select $\mathrm{CAV}_{t_{\text {start }}: t_{\text {end }}}$ with $t_{\text {start }}$ being 24 hours before AKI to evaluate $t C A$ and $C S$ scores.

Presence of the concept over time: When using a 24-hour sliding window of activation differences, we obtain higher $t C A$ scores directly before the AKI event $\left(t_{1}, 2\right.$ hours before AKI) when the

\footnotetext{
${ }^{2}$ Given that the end point (AKI 1+) is controlled for, $\mathrm{CAV}_{\boldsymbol{t}_{\text {end }}}$ is not built.
} 
a

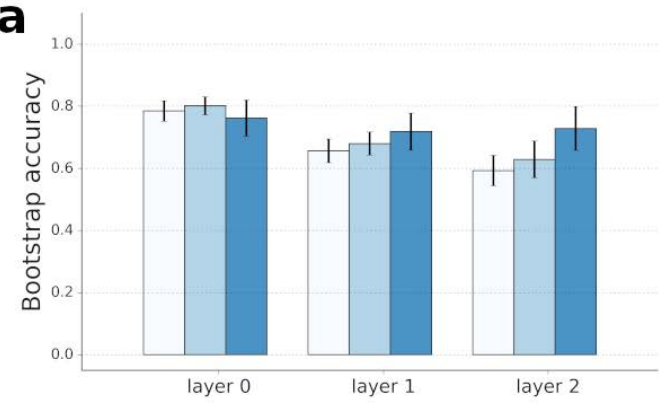

b

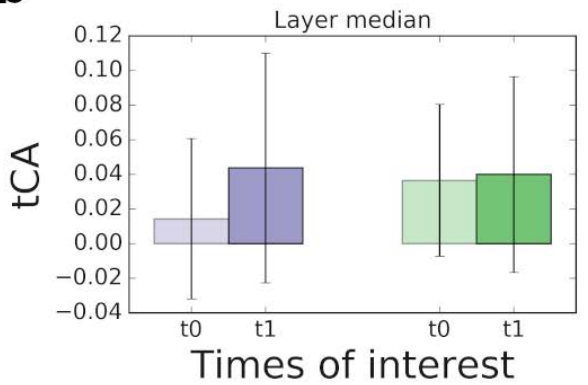

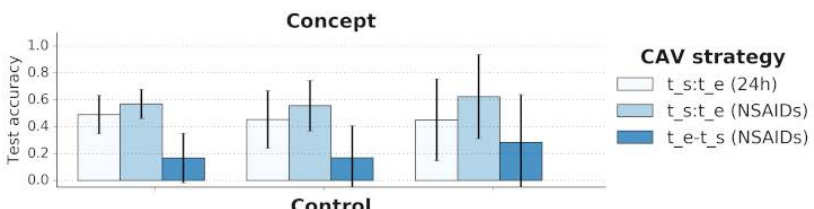

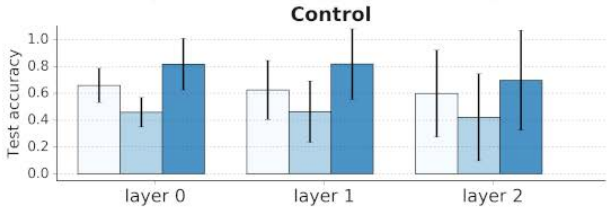

C

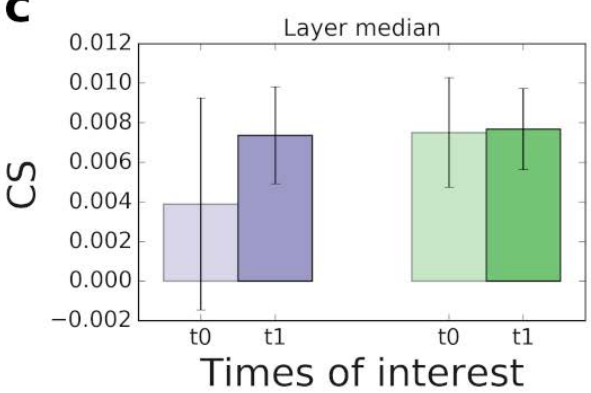

$\square$ Concept

Concept

Control

Control
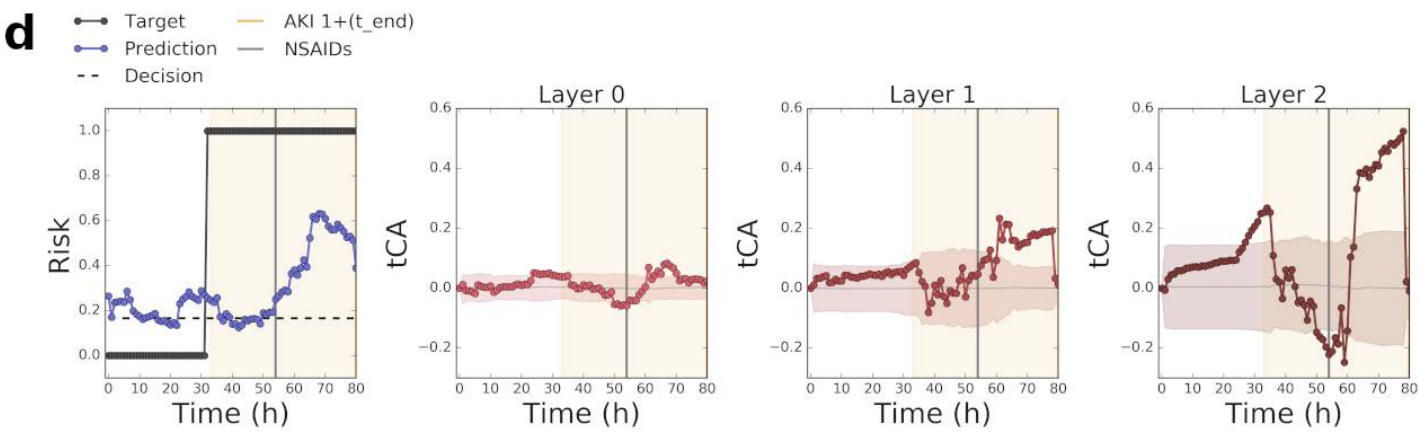

Figure 4: MIMIC results, NSAIDs concept. a CAV linear model performance on held-out samples (left) and on test samples for concept (top) and control (bottom) samples. Each bar represents a CAV building strategy. $b t C A$ global scores, averaged across patients (mean \pm std) for the concept (purple) and control (green) groups 48 hours before AKI $\left(t_{0}\right)$ and at time of AKI stage $2\left(t_{1}\right)$. c $C S$ scores. $d$ Single patient timeseries, displaying the label (AKI 1+ within 48h) and model's output, as well as $t C A$ for each layer, and its null hypothesis (shaded). The yellow shaded area represents the prediction horizon of the model, i.e. within 48 hours of AKI 1+. The administration of NSAIDs is displayed by a grey vertical line.

concept is present compared to at time of NSAIDs ( $t_{0}$, Figure $4 \mathrm{~b}$ ). This difference is however small and similar scores are obtained close to the AKI endpoint on control patients. Figure $4 \mathrm{~d}$ displays an example trajectory from the evaluation set for $\mathrm{CAV}_{t_{\text {start }}: t_{\text {end }}}$. We discern an increase in alignment, outside of the $\pm 1 \times$ standard deviation, after the time of NSAIDs administration on the three layers. See the Supplement for more positive local examples. We observe no or negative alignment for negative predictions (Figure 5, a,b). On the other hand, we observe an increase in alignment at time of NSAIDs administration, simultaneous to a false positive prediction (around $t=40$, Figure $5 \mathrm{c}$ ).

Influence of the $C A V$ :. Consistent with our observations, CS displays a small effect of the concept on the predictions at the time of AKI, but the pattern is not as clear as for the AKI concept (Figure 4c).
This could reflect either that the CAV does not properly represent the NSAIDs direction, or that the model is not only marginally influenced by this direction when making predictions. Further work will investigate other nephrotoxic agents as well as involve a clinical evaluation of patients to ensure that the agent caused the AKI.

\subsubsection{Antibiotics concept.}

Building the $C A V$ :. Given previous results, we select $\mathrm{CAV}_{t_{\text {start }}: t_{\text {end }}}$ with $t_{\text {start }}$ being 24 hours before AKI to build CAVs and evaluate $t C A$ and $C S$ scores. The classifiers are assessed as significant per non-parametric permutation testing, with accuracies of $61.04 \%$, $64.77 \%$ and $63.38 \%$ and ROC AUC of $0.6891,0.7275$ and 0.7325 for layers 1,2 and 3 , respectively $(p<0.001)$. 
$\mathbf{a}$

$$
\begin{aligned}
& \because \text { Target }- \text { AKI } 1+(\text { t_end }) \\
& \because \text { Prediction }- \text { NSAIDs } \\
& -- \text { Decision }
\end{aligned}
$$
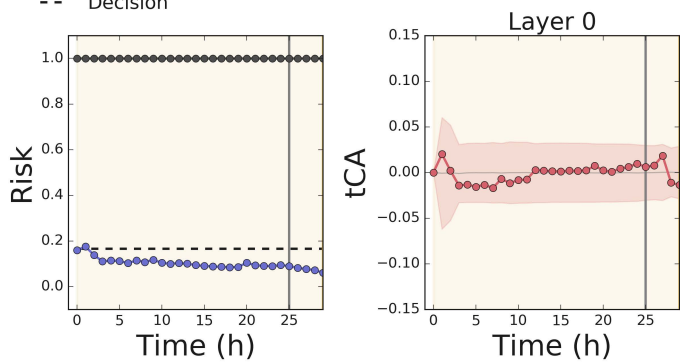

b

$$
\begin{aligned}
& \longrightarrow \text { Target }- \text { AKI 1+(t_end) } \\
& \because \text { Prediction }- \text { NSAIDs } \\
& - \text { Decision }
\end{aligned}
$$

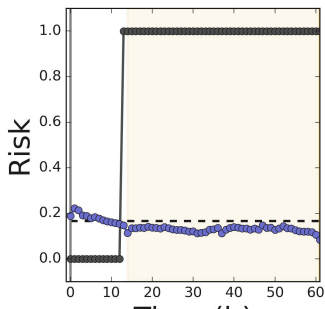

Time (h)

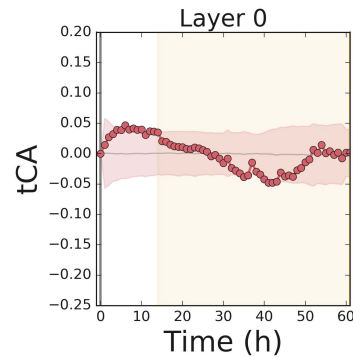

c

$$
\begin{aligned}
& \because \text { Target }- \text { AKI 1+(t_end) } \\
& \because \text { Prediction }- \text { NSAIDs } \\
& \because \text { Decision }
\end{aligned}
$$
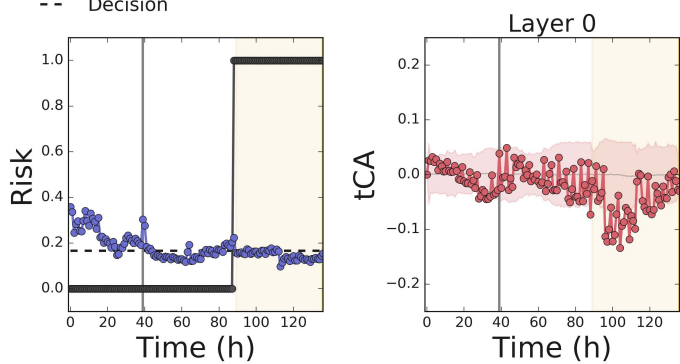
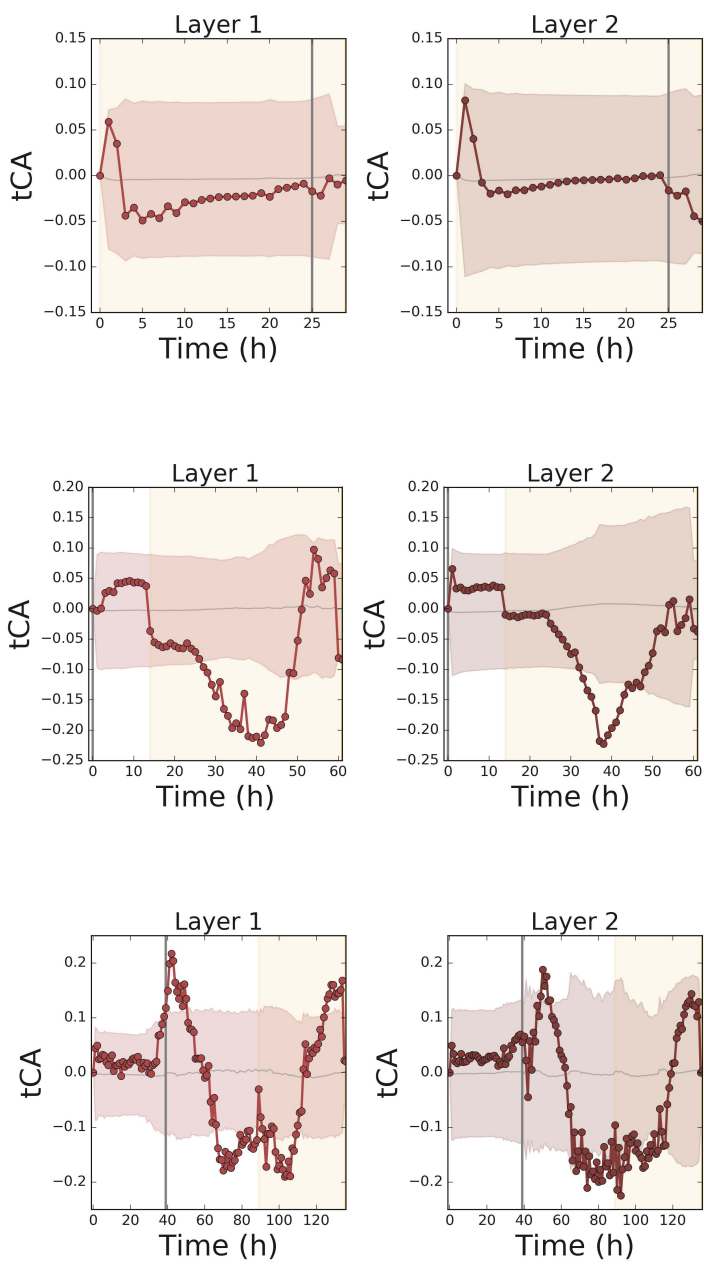

Figure 5: Local MIMIC results, NSAIDs concept. a,b Negative predictions, c false positive prediction at time of NSAIDs. Similar to Figure 4d.

Presence of the concept over time: When using a 24-hour sliding window of activation differences, we obtain higher $t C A$ scores at $t_{1}$ when the concept is present compared to at time $t_{\text {start }}$ (Figure 6). As for the NSAIDs concept, we observe increases in local tCA scores when the concept is present (Figure 6 and Supplementary).

Influence of the $C A V$ :. As per CS, the 'antibiotics' concept has an influence on AKI predictions (Figure $6 \mathrm{~b}$ ).

3.2.5 Sex concept. None of the considered strategies to build CAVs leads to significant results, with balanced accuracy ranging between 0.4727 and $0.5905(p=0.19)$, and ROC AUC between 0.4516 and 0.6190 ( $p=0.18$, see Supplement for details). Interestingly, model performance on subgroups display no striking imbalance between male and female subgroups (0.5953 PRAUC for females and 0.5809 for males, with ROCAUC of 0.8624 and 0.8445 respectively). This result could suggest that features related to $\mathrm{AKI}$ are not significantly different between sexes, or that the model does not encode sex to predict AKI using the MIMIC benchmark dataset. On the other hand, non-significant CAVs could also arise from technical factors such as, e.g. the patient selection being too heterogeneous, or the time window selected for CAV building not including the feature changes between sexes. Therefore, a non-significant CAV does not allow to conclude that the model is not relying on the considered signal.

3.2.6 Comparison with feature-based attributions. Table 1 displays the top 15 features ranked by their attribution scores, computed from the test set. We observe little overlap between the two lists ${ }^{3}$, apart from Serum urea nitrogen and Serum creatinine. While it is reassuring to see these features in both rankings given their direct relationship to the definition of the AKI label [14], they do not represent the 'cause' of the adverse event, and are hence not 'actionable' from a clinical perspective. Interestingly, the ranking for

\footnotetext{
${ }^{3}$ Please note that the behavior of gradient and occlusion attribution techniques under feature heterogeneity (i.e. binary vs numerical), as well as sparsity, has not been extensively investigated and the results presented here could be affected by those factors.
} 
a

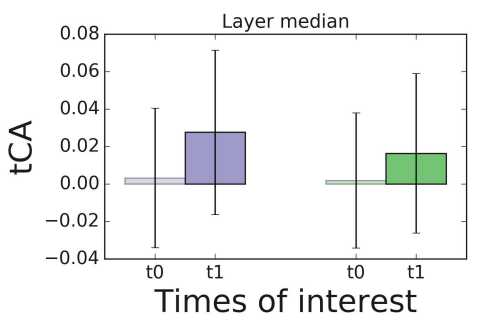

b

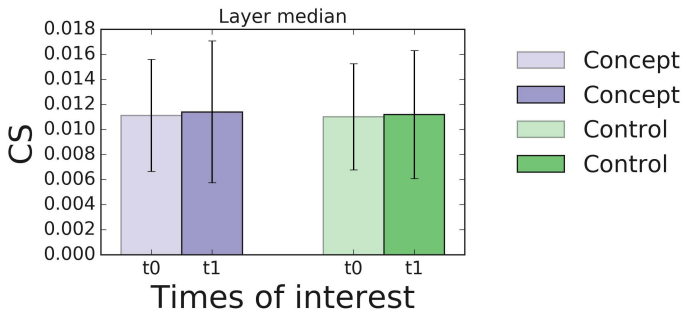

c
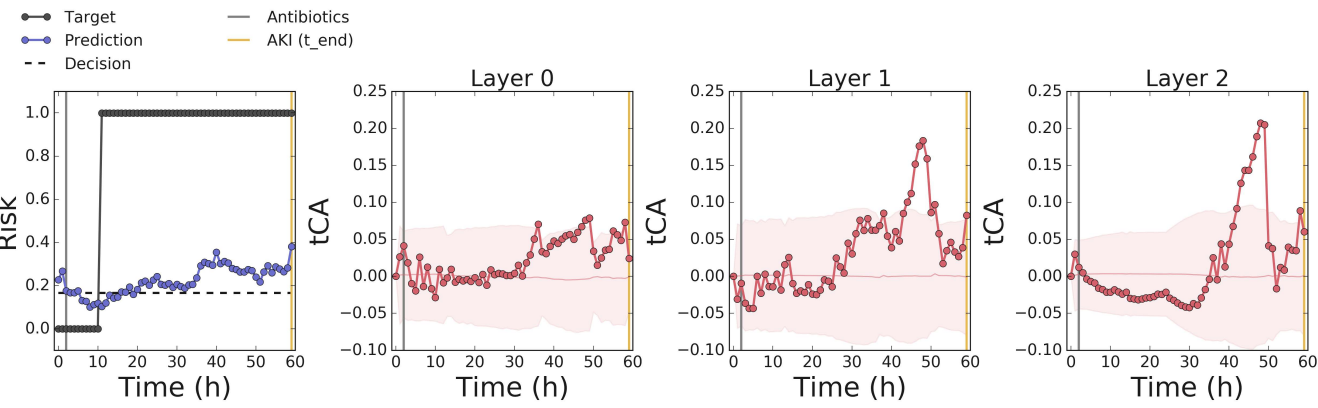

Figure 6: MIMIC results, Antibiotics concept. tCA (a) and CS (b) results at time of antibiotics, and at time of AKI. c Local patient trajectory with time of antibiotics displayed as a vertical grey line.

gradients highlights an antibiotic (Nafcillin), used in our definition of the 'Antibiotics' concept.

For the patient presented in Figure 6, the predicted risk at time of antibiotics is low, and the model does not include this feature in its top 10 according to occlusion. Gradients however display Vancomycin, as well as heart rate and respiratory rate in the top 10 , features that can be related to microbial infections. We see in Figure $6 \mathrm{c}$ that the alignment with the model's prediction does not happen at the time of antibiotics, but closer to the AKI event, highlighting the fact that TCAV alignment scores are not acting as 'feature detectors'.

\section{DISCUSSION AND FUTURE WORK}

In this work, we explore the use of TCAV for RNNs, by defining concepts as trajectories over time. Across datasets and concepts, we notice that $\mathrm{CAV}_{t_{\text {start }}: t_{\text {end }}}$ consistently leads to better generalization across time points. This result suggests that a majority of time steps in the time windows selected were relevant for the considered concepts. We note that extending this window or increasing the variability of the signals within this window might lead to different results. Nevertheless, we show that this approach provides meaningful CAVs and that both $t C A$ and CS scores are consistent with our expectations on the synthetic data. We observe that $t C A$ computed as a temporal "derivative" saturates if the concept's presence or absence does not vary over the time window $[t-d t, t]$. Similarly, CS highlights transitions in the model's predictions when taking the gradients w.r.t. the sigmoid of the logits, as the sigmoid saturates when the prediction is further away from the decision boundary. This could suggest a better use case of $t C A$ and CS in alert-based settings, where predictions/explanations are provided at specific time points, e.g. when the predicted risk passes the decision threshold.
This work focuses on explaining predictions from RNNs and is hence bound by the model architecture. Recent works have however investigated other architectures for EHR predictions, including transformers [27] and point-wise convolutions [21]. While RNNs are adapted to the large number of features considered in the present model (tens of thousands compared to a couple of hundreds or less in newer architectures), future work could investigate how the approach developed here could be applied to other architectures.

While our approach factors in temporality in the construction of CAVs and $t C A$, CS would need to be extended to be able to account for how differences in model predictions relate to changes in the presence/absence of a concept over time. In this regard, a potential direction of work would be to refer to Temporal Integrated Gradients [9]. Such a method based on integrated gradients [28] would also enable the use of the proposed approach for local explanations, as integrated gradients estimate the difference between the obtained prediction and a "neutral decision".

One limitation of TCAV arises from the difficulty of defining a concept through examples from real-world EHR data. While toy datasets or ImageNet applications seem intuitive, healthcare data can be difficult to separate into clinical concepts. In the present work, we computed relative CAVs by selecting concept and control examples using simple rules, typically based on one ('sex') or an ensemble of features. Our results suggest that the proposed approach can capture correlated signals and trajectories based on this proxy definition, and do not act as 'feature detectors'. Based on their definition, concepts can be defined to represent 'actionable' clinical concepts and can investigate signals that would not be directly represented as features in the model training set (e.g. gender). This is in contrast to feature-based attributions, that are tied to the features present in the samples used to compute attributions. However, concepts, while designed to be human-understandable, will typically encompass multiple features, as well as potential changes 
Occlusion

\begin{tabular}{lll}
\hline Feature type & Score & Feature Name \\
\hline Vitals, presence & 0.0193 & Bair hugger \\
Vitals, presence & 0.0177 & Nitric Oxide PPM \\
Vitals, presence & 0.0176 & Nitric tank pressure \\
Labs, presence & 0.0132 & Serum urea nitrogen \\
Vitals, presence & 0.0127 & Dialysis Access Type: catheter \\
Vitals, presence & 0.0120 & Dialysis Type: peritoneal \\
Vitals, presence & 0.0116 & Emesis [Appearance] \\
Vitals, presence & 0.0114 & GI Tube Place Method: gastric ph \\
Labs, presence & 0.0103 & Serum creatinine \\
Vitals, presence & 0.0098 & Diagnosis/op: hyperglycemia \\
Vitals, presence & 0.0093 & Dialysis Access Site: abdomen \\
Labs, value & 0.0087 & Serum urea nitrogen \\
Vitals, presence & 0.0085 & PA Catheter Waveform Appear: unable \\
& & to wedge \\
Vitals, presence & 0.0080 & Allergy 1: thiazides \\
Vitals, presence & 0.0077 & Micro-Neb Treatment: alb/neb
\end{tabular}

Gradients

\begin{tabular}{lll}
\hline Feature type & Score & Feature Name \\
\hline Prescription, presence & 0.0148 & Nephrocaps \\
Prescription, presence & 0.0042 & Sulfameth/Trimethoprim (IV) \\
Admission, domain & 0.0038 & - \\
Labs, value & 0.0036 & Serum creatinine \\
Prescription, presence & 0.0035 & Nafcillin (IV) \\
Vitals, presence & 0.0034 & System Integrity: clots present \\
Prescription, presence & 0.0033 & Furosemide \\
Vitals, presence & 0.0030 & Heparin Dose (per hour) \\
Prescription, presence & 0.0030 & Atropine Sulfate (IV) \\
Labs, value & 0.0026 & tbili \\
Prescription, presence & 0.0025 & NS Epidural Bag \\
Prescription, presence & 0.0023 & Midodrine HCl \\
Vitals, presence & 0.0021 & Impaired Skin: extremities, lo \\
& & \\
Prescription, presence & 0.0018 & Heparin (Hemodialysis) \\
Prescription, presence & 0.0017 & Nitroglycerin (IV drip)
\end{tabular}

Table 1: Occlusion and gradient attribution scores, averaged across patients and time steps for global explanations.

\begin{tabular}{lll} 
Occlusion & & Gradients \\
${$\cline { 1 - 1 }$} }$ & & Lorazepam, pres \\
Potassium Chloride, pres & & Heart Rate, value \\
Syringe (Neonatal), value & & Vancomycin, pres \\
Noninvasive Systolic, value & & Safety Measures, family, pres \\
Normocarb, pres & Calcium Gluconate, value \\
Potassium Chloride, value & Lactulose, pres \\
Docusate Sodium, pres & Respiratory Rate, value \\
Sodium Fluoride, pres & Soln., pres \\
Sodium Fluoride, value & D5W, pres \\
Urine Output, value & Senna, pres
\end{tabular}

Table 2: Occlusion and gradient attribution scores, at time of antibiotics for the patient presented in Figure6.

in patterns of those features across time. Therefore, feature-based attributions could be used in conjunction with concept-based attributions to understand which specific features could affect the model's alignment with a concept, e.g. which physiological features have been affected by the microbial infection as treated by the antibiotics.

We further matched control examples for a number of criteria. We note that other matching criteria and methods could be used, e.g. propensity score matching or other optimal transport techniques. While we reported promising results on multiple concepts using these CAVs, a danger is to miss confounding factors that then lead to a significant CAV. A future direction could be to generate counterfactuals, as in $[8,20,26]$. Given the dimensionality of the data, training and evaluating such a counterfactual generative model however remains challenging. Another risk lies in potential confirmation bias during the process of building the CAV and estimating $t C A$ and CS scores, as the user might be tuning the CAV building until a concept surfaces. It could hence be desirable to know how much signal is covered by a set of concepts, as proposed in [32]. On the other hand, the clinical user might want to define a limited set of "actionable" concepts, e.g. "dehydration" or "nephrotoxicity", for which a clinical action could prevent the predicted outcome.
This would alleviate the concerns around building the "complete" set of concepts, and provide a path to action, especially in the case of local explanations. We also note that defining concepts requires the involvement of clinicians. We believe that this is a strength of the method rather than a weakness, as it allows clinicians to define what "actionable" or "trustworthy" mean in the selected use case, leading to increased transparency in the machine learning development pipeline.

Finally, we evaluate the proposed approach empirically, based on the 'ground truth' present in the synthetic data, as well as on a clinical benchmark. Our concepts and results for MIMIC were assessed by a clinician. Future work should however assess the clinical relevance and utility of TCAV for EHR more rigorously, using human and task grounded evaluations, as suggested in [4]. In particular, it would be useful to investigate whether concept-based explanations can help guide clinical actions taken in response to a prediction, and, ultimately, whether these explanations improve outcomes for patients.

\section{SOFTWARE AND DATA}

The Python and TensorFlow code to generate the synthetic dataset, models, and compute CS and $t C A$ is available on Github at https: //github.com/google/ehr-predictions/tree/master/tcav-for-ehr. The de-identified EHR data is available based on a user agreement at https://physionet.org/content/mimiciii/1.4/.

\section{ACKNOWLEDGMENTS}

We thank Been Kim and Yash Goyal for discussions and for sharing code.

\section{REFERENCES}

[1] R Ambrosino, B G Buchanan, G F Cooper, and M J Fine. 1995. The use of misclassification costs to learn rule-based decision support models for cost-effective hospital admission strategies. Proceedings. Symposium on Computer Applications in Medical Care (1995), 304-8. http://www.ncbi.nlm.nih.gov/pubmed/8563290http: //www.pubmedcentral.nih.gov/articlerender.fcgi?artid=PMC2579104 
[2] Rich Caruana, Yin Lou, Johannes Gehrke, Paul Koch, Marc Sturm, and Noemie Elhadad. 2015. Intelligible Models for HealthCare. In Proceedings of the 21th ACM SIGKDD International Conference on Knowledge Discovery and Data Mining - KDD '15. 1721-1730. https://doi.org/10.1145/2783258.2788613

[3] Edward Choi, Mohammad Taha Bahadori, Joshua A. Kulas, Andy Schuetz, Walter F. Stewart, and Jimeng Sun. 2016. RETAIN: An interpretable predictive model for healthcare using reverse time attention mechanism. In Advances in Neural Information Processing Systems. Neural information processing systems foundation, 3512-3520. arXiv:1608.05745

[4] Finale Doshi-Velez and Been Kim. 2018. Considerations for Evaluation and Generalization in Interpretable Machine Learning. 3-17. https://doi.org/10.1007/ 978-3-319-98131-4 1

[5] Marine Flechet, Stefano Falini, Claudia Bonetti, Fabian Güiza, Miet Schetz, Greet Van den Berghe, and Geert Meyfroidt. 2019. Machine learning versus physicians' prediction of acute kidney injury in critically ill adults: a prospective evaluation of the AKIpredictor. Critical Care 23, 1 (16 Aug 2019), 282. https://doi.org/10. 1186/s13054-019-2563-x

[6] Joseph Futoma, Sanjay Hariharan, Katherine Heller, Mark Sendak, Nathan Brajer Meredith Clement, Armando Bedoya, and Cara O'brien. 2017. An Improved MultiOutput Gaussian Process RNN with Real-Time Validation for Early Sepsis Detection. Technical Report. arXiv:1708.05894v1

[7] A. L. Goldberger, L. A. Amaral, L. Glass, J. M. Hausdorff, P. C. Ivanov, R. G. Mark, J. E. Mietus, G. B. Moody, C. K. Peng, and H. E. Stanley. 2000. PhysioBank, PhysioToolkit, and PhysioNet: components of a new research resource for complex physiologic signals. Circulation 101, 23 (2000). https://doi.org/10.1161/01.cir.101. 23.e215

[8] Yash Goyal, Uri Shalit, and Been Kim. 2019. Explaining Classifiers with Causal Concept Effect (CaCE). (jul 2019). arXiv:1907.07165 http://arxiv.org/abs/1907. 07165

[9] Michaela Hardt, Alvin Rajkomar, Gerardo Flores, Andrew Dai, Michael Howell, Greg Corrado, Claire Cui, and Moritz Hardt. 2019. Explaining an increase in predicted risk for clinical alerts. Technical Report. arXiv:1907.04911v1 https: //arxiv.org/abs/1907.04911

[10] Sepp Hochreiter and Juergen Schmidhuber. 1997. Long Short-Term Memory. Neural Computation 9, 8 (1997), 1735-1780. http://www7.informatik.tumuenchen.de/\{ $\}$ hochreithttp://www.idsia.ch/\{ \}juergen

[11] Alistair E.W. Johnson, Tom J Pollard, and Roger G. Mark. 2016. MIMIC-III Clinical Database. https://doi.org/10.13026/C2XW26

[12] Alistair E.W. Johnson, Tom J. Pollard, Lu Shen, Li Wei H. Lehman, Mengling Feng, Mohammad Ghassemi, Benjamin Moody, Peter Szolovits, Leo Anthony Celi, and Roger G. Mark. 2016. MIMIC-III, a freely accessible critical care database. Scientific Data 3 (may 2016). https://doi.org/10.1038/sdata.2016.35

[13] Rohit J. Kate, Noah Pearce, Debesh Mazumdar, and Vani Nilakantan. 2020. A continual prediction model for inpatient acute kidney injury. Computers in Biology and Medicine 116 (2020), 103580. https://doi.org/10.1016/j.compbiomed. 2019.103580

[14] Arif Khwaja. 2012. KDIGO clinical practice guidelines for acute kidney injury. https://doi.org/10.1159/000339789

[15] Been Kim, Martin Wattenberg, Justin Gilmer, Carrie Cai, James Wexler, Fernanda Viegas, and Rory Sayres. 2018. Interpretability beyond feature attribution: Quantitative Testing with Concept Activation Vectors (TCAV). In 35th International Conference on Machine Learning, ICML 2018, Vol. 6. 4186-4195. arXiv:1711.11279 http://arxiv.org/abs/1711.11279

[16] Zachary C. Lipton, David C. Kale, Charles Elkan, and Randall Wetzel. 2016. Learning to diagnose with LSTM recurrent neural networks. In 4th International Conference on Learning Representations, ICLR 2016 - Conference Track Proceedings. International Conference on Learning Representations, ICLR. arXiv:1511.03677

[17] James Munkres. 1957. Algorithms for the Assignment and Transportation Problems. F. Soc. Indust. Appl. Math. 5, 1 (mar 1957), 32-38. https://doi.org/10.1137/ 0105003

[18] Cecilia Panigutti, Alan Perotti, Dino Pedreschi, and Dino 2020 Pedreschi. 2020. An ontology-based approach to black-box sequential data classification explanations. (2020). https://doi.org/10.1145/3351095.3372855

[19] Seyedeh Neelufar Payrovnaziri, Zhaoyi Chen, Pablo Rengifo-Moreno, Tim Miller Jiang Bian, Jonathan H Chen, Xiuwen Liu, and Zhe He. 2020. Explainable artificial intelligence models using real-world electronic health record data: a systematic scoping review. Fournal of the American Medical Informatics Association (2020). https://doi.org/10.1093/jamia/ocaa053

[20] Stephen Pfohl, Tony Duan, Daisy Yi Ding, and Nigam H Shah. 2019. Counterfactual Reasoning for Fair Clinical Risk Prediction. In Proceedings of Machine Learning Research, Vol. 85. 1-29. arXiv:1907.06260v1

[21] Emma Rocheteau, Pietro Liò, and Stephanie Hyland. 2020. Temporal Pointwise Convolutional Networks for Length of Stay Prediction in the Intensive Care Unit arXiv (jul 2020). arXiv:2007.09483 http://arxiv.org/abs/2007.09483

[22] Ying Sha and May D. Wang. 2017. Interpretable predictions of clinical outcomes with an attention-based recurrent neural network. In ACM-BCB 2017 - Proceedings of the 8th ACM International Conference on Bioinformatics, Computational Biology, and Health Informatics. Association for Computing Machinery, Inc, New York,
NY, USA, 233-240. https://doi.org/10.1145/3107411.3107445

[23] Benjamin Shickel, Tyler J. Loftus, Lasith Adhikari, Tezcan Ozrazgat-Baslanti, Azra Bihorac, and Parisa Rashidi. 2019. DeepSOFA: A Continuous Acuity Score for Critically Ill Patients using Clinically Interpretable Deep Learning. Scientific Reports 9, 1 (dec 2019). https://doi.org/10.1038/s41598-019-38491-0

[24] Michael Simonov, Ugochukwu Ugwuowo, Erica Moreira, Yu Yamamoto, Aditya Biswas, Melissa Martin, Jeffrey Testani, and F. Perry Wilson. 2019. A simple real-time model for predicting acute kidney injury in hospitalized patients in the US: A descriptive modeling study. PLOS Medicine 16, 7 (07 2019), 1-15. https://doi.org/10.1371/journal.pmed.1002861

[25] Karen Simonyan, Andrea Vedaldi, and Andrew Zisserman. 2013. Deep Inside Convolutional Networks: Visualising Image Classification Models and Saliency Maps. (2013). arXiv:1312.6034 http://arxiv.org/abs/1312.6034

[26] Sumedha Singla, Brian Pollack, Junxiang Chen, and Kayhan Batmanghelich. 2019. Explanation by Progressive Exaggeration. (2019). arXiv:1911.00483 http: //arxiv.org/abs/1911.00483

[27] Huan Song, Deepta Rajan, Jayaraman J. Thiagarajan, and Andreas Spanias. 2017. Attend and diagnose: Clinical time series analysis using attention models. In 32nd AAAI Conference on Artificial Intelligence, AAAI 2018. AAAI press, 4091-4098. https://asu.pure.elsevier.com/en/publications/attend-and-diagnoseclinical-time-series-analysis-using-attention

[28] Mukund Sundararajan, Ankur Taly, and Qiqi Yan. 2017. Axiomatic attribution for deep networks. In 34th International Conference on Machine Learning, ICML 2017, Vol. 7. 5109-5118. arXiv:1703.01365

[29] Nenad Tomašev, Xavier Glorot, Jack W. Rae, Michal Zielinski, Harry Askham, Andre Saraiva, Anne Mottram, Clemens Meyer, Suman Ravuri, Ivan Protsyuk, Alistair Connell, Cían O. Hughes, Alan Karthikesalingam, Julien Cornebise, Hugh Montgomery, Geraint Rees, Chris Laing, Clifton R. Baker, Kelly Peterson, Ruth Reeves, Demis Hassabis, Dominic King, Mustafa Suleyman, Trevor Back, Christopher Nielson, Joseph R. Ledsam, and Shakir Mohamed. 2019. A clinically applicable approach to continuous prediction of future acute kidney injury. Nature 572, 7767 (aug 2019), 116-119. https://doi.org/10.1038/s41586-019-1390-1

[30] Sana Tonekaboni, Shalmali Joshi, Melissa D McCradden, and Anna Goldenberg. 2019. What Clinicians Want: Contextualizing Explainable Machine Learning for Clinical End Use. In Proceedings of Machine Learning Research. 1 - 21. arXiv:1905.05134 http://arxiv.org/abs/1905.05134

[31] Cao Xiao, Edward Choi, and Jimeng Sun. 2018. Opportunities and challenges in developing deep learning models using electronic health records data: A systematic review. Fournal of the American Medical Informatics Association 25, 10 (2018), 1419-1428. https://doi.org/10.1093/jamia/ocy068

[32] Chih-Kuan Yeh, Been Kim, Sercan O. Arik, Chun-Liang Li, Pradeep Ravikumar, and Tomas Pfister. 2019. On Concept-Based Explanations in Deep Neural Networks. In Proceedings of the 33rd Conference on Neural Information Processing Systems (NeurIPS 2019). arXiv:1910.07969 http://arxiv.org/abs/1910.07969

[33] Matthew D. Zeiler and Rob Fergus. 2014. Visualizing and understanding convolutional networks. In Lecture Notes in Computer Science (including subseries Lecture Notes in Artificial Intelligence and Lecture Notes in Bioinformatics), Vol. 8689 LNCS. 818-833. https://doi.org/10.1007/978-3-319-10590-1_53 